\title{
Proposal of industrialization process of dredged sediments
}

\section{Proposition d'un processus d'industrialisation des sédiments de dragage}

\author{
Hadj Sadok Rachid ${ }^{1}$, Belas Nadia ${ }^{1}$, Tahlaiti Mahfoud ${ }^{2}$, Belaribi Omar ${ }^{1}$, Taieb Fatima ${ }^{1}$, Belguesmia Khalil ${ }^{1}$, Safer Omar $^{1}$ \\ ${ }^{1}$ Laboratoire Construction Transport et Protection de l'Environnement, Université de Mostaganem, Département génie \\ civil et architecture, Mostaganem, Algérie \\ ${ }^{2}$ Laboratoire de l'institut de recherche en génie civil et mécanique (GeM) et l'institut catholique d'art et des métiers \\ (ICAM) à Nantes, Ecole centrale de Nantes, France
}

\begin{abstract}
Our study will focus on life cycle assessment (LCA) of dredged sediments in its environment from sediment extraction to waste treatment. This tool is part of an environmental management approach, to compare the environmental loads of the different stages of the life cycle of the same product and to deduce which stage of the scenario is the most polluting in environmental terms. Optimize the modeling of industrialization process of dredged sediments using the SimaPro 8.2.3 software to propose a model that is the most respectful of the environment. We will focus on the environmental impacts; we will try to propose the most environmentally friendly scenario to exploit these dredged sediments in the field of building construction.
\end{abstract}

\begin{abstract}
Résumé. Notre étude portera essentiellement sur l'analyse de cycle de vie (ACV) des sédiments dragués dans son environnement depuis l'extraction des sédiments jusqu'au traitement des déchets. Cet outil s'inscrit dans une démarche de management environnemental, pour comparer les charges environnementales des différentes étapes du cycle de vie du même produit; et en déduire quelle étape du scenario est la plus polluante en termes environnementaux. Optimiser la modélisation d'un processus d'industrialisation des sédiments de dragage à l'aide du logiciel SimaPro 8.2.3 pour proposer un modèle le plus respectueux de l'environnement. On se focalisera sur les impacts environnementaux ; On essaiera de proposer le scénario le plus respectueux de l'environnement, afin d'exploiter ces sédiments dragués dans le domaine de la construction des bâtiments.
\end{abstract}

\section{Introduction}

Cet article s'inscrit dans une longue étude de recherche au sein du laboratoire LCTPE portant sur le domaine de la valorisation des sédiments de dragage. Dans cette étape de recherche, on complètera le travail par une étude environnementale par analyse du cycle de vie des sédiments de dragage, pour faire face au problème d'envasement des barrages dû à l'érosion hydraulique mettant en danger ses potentialités. L'Algérie a investi des moyens colossaux pour alimenter en eau potable sa population et permettre l'irrigation des terres agricoles. De grands efforts ont été déployés pour assurer l'autosuffisance en eau potable et fournir l'eau aux industriels ; Des réalisations de barrages de toutes dimensions à travers tout le territoire national ; ont vu et vont pouvoir voir le jour.

Plusieurs études de recherches récentes $[1][2][3][4][5][6][7]$ sur la valorisation des sédiments issus du dragage des barrages ont été menées dans le domaine de caractérisation physico-chimique, mécanique et de durabilité. Elles ont démontré la possibilité de leur utilisation en tant que matière première dans le domaine de génie civil, et spécialement en substitution partielle des sédiments de dragage dans le ciment.

L'Algérie dispose de 72 grands barrages, d'une capacité totale de 7692,24 millions $\mathrm{m}^{3}$ selon les dernières mesures en 2014. Leur envasement est d'environ 1085,94 millions $\mathrm{m}^{3}$. Cela représente un pourcentage d'envasement de 14,12\% de la capacité totale [8].

De grandes opérations de dragage essaient d'éliminer une partie des sédiments dès leur arrivée dans les réservoirs des barrages. La troisième campagne de dévasement est en cours depuis 2014. Trois grandes opérations de dragage des barrages de Bouhanifia et Foum El Gherza et K'sob sont en cours. Elles visent à récupérer 21,3 millions $\mathrm{m}^{3}$ de capacité de stockage dans un délai moyen 
de 30 mois [8].

\section{La description du système de production des sédiments de dragage}

Dans cette partie d'industrialisation des sédiments de dragage, nous allons détailler le procédé de production et décrire les différents processus de traitement des sédiments; afin de les valoriser comme matière première dans la substitution du ciment. Pour cela deux scenarios sont envisageables :

\subsection{Scénario $\left(\mathrm{SN}^{\circ} 1\right)$}

Il consiste à utiliser les sédiments après la décantation totale et le séchage à l'air libre dans les zones de rejet. Là on extrait ces sédiments par un chargeur et on les transporte avec un dumper jusqu'à la station de traitement des sédiments de dragage ; l'installation de l'usine étant au niveau des zones de rejet.

\subsection{Scénario ( $\left.\mathrm{SN}^{\circ} 2\right)$}

On exploite les sédiments après dragage dans les zones de rejets ou au niveau des conduites terrestres, est incorporé un sécheur directement pour récupérer l'eau et les sédiments séchés, afin d'optimiser le temps de décantation à l'air libre. Le temps de séjour sera de 10 à $60 \mathrm{~min}$, avec une consommation énergétique réduite 0,6$0,7 \mathrm{kWh} / \mathrm{kg}$ d'eau évaporée. On suppose que le taux d'humidité est de $30 \%$ pour le scénario $\mathrm{N}^{\circ} 2$ [9].

\section{Méthodologie de l'analyse du cycle de vie}

L'analyse de cycle de vie (ACV) est la méthodologie de référence la plus complète pour réaliser l'inventaire des flux quantifies et évaluer les impacts environnementaux d'un produit ou service; Elle distingue 4 étapes [10] :

a) La définition des frontières du système étudié, de l'unité fonctionnelle et du périmètre prise des informations et des données.

b) La réalisation de l'inventaire du cycle de vie à collecter et compiler.

c) La transformation de cet inventaire sous forme d'indicateur des impacts.

d) L'interprétation des résultats.

\section{L'analyse du cycle de vie de production des sédiments de dragage}

\subsection{Le champ de l'étude (Scope)}

\subsubsection{Fonction}

L'analyse portera sur les quatre étapes de production, le séchage, le concassage, la calcination et le stockage des sédiments de dragage. Il s'agira de modéliser les différentes étapes de production afin de produire une tonne de sédiments de dragage calcinés prêts à être substituée dans le ciment.

\subsubsection{Unité fonctionnelle}

Production d'une (1) tonne de sédiments de dragage calcinés pour la substitution partielle dans le ciment (pour une finesse $\geq 3000 \mathrm{~cm}^{2} / \mathrm{g}$ ).

\subsubsection{Hypothèses et limitations}

Pour toute cette analyse de processus de production des sédiments de dragage, on exclut l'étape de dragage ; car cette étape est faite par l'ANBT [8]. On exploite les sédiments après dragage dans les zones de rejet.

Pour les différents procédés de production, référence est faite aux données collectées au niveau de la recherche bibliographique effectuée dans ce travail sur la valorisation des sédiments de dragage. Nous allons détailler toutes ces données dans l'inventaire du cycle de vie.

La fin de vie n'a pas été prise en compte dans cette analyse car l'objectif final de cette recherche est l'application de l'analyse du cycle de vie des sédiments de dragage dans le domaine bâtiment.

\subsubsection{Frontière du système}

On va calculer les flux élémentaires entrants dans le processus de production des sédiments de dragage, comme l'utilisation des ressources naturelles et l'énergie ; ainsi que l'utilisation d'espace au niveau des zones de rejet. Les flux élémentaires sortants dans l'environnement sont des émissions dans l'air, l'eau et le sol.

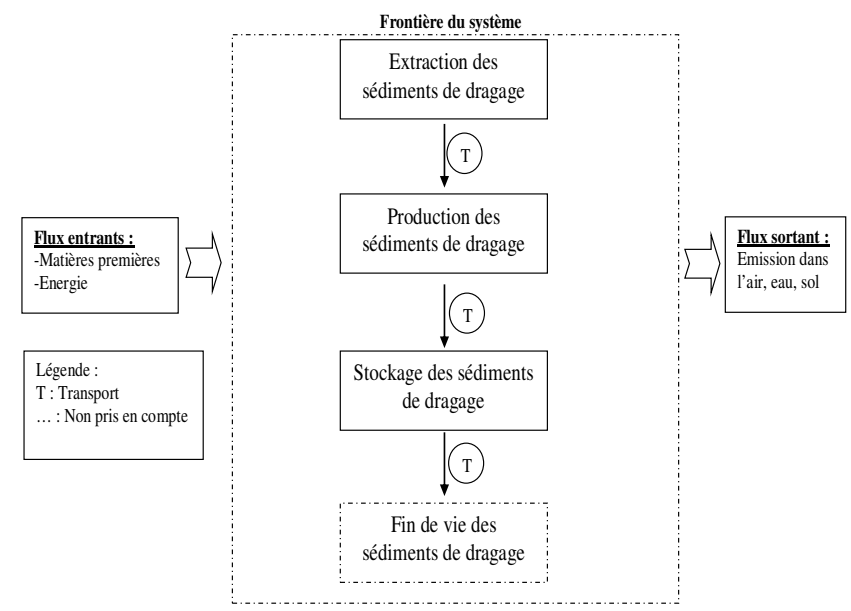

Fig. 1. Principe de définition de la frontière du système

\subsection{L'inventaire du cycle de vie :}

4.2.1 La méthode de collecte de données:

Les données ont été collectées on deux étapes :

1) Une recherche bibliographique sur la valorisation des sédiments de dragage. 
2) L'exploitation de la base de données Ecoinvent par le logiciel SimaPro 8.2.3 multi-user de l'école d'ingénieurs ICAM de Nantes.

4.2.2 L'inventaire du procédé de production des sédiments de dragage

Il est donné par le tableau 1

Table 1. Inventaire du procédé de production des sédiments de dragage

\begin{tabular}{|c|c|c|c|c|c|}
\hline Procédé & Consommation & Unité & Scénario N01 & Scénario $\mathrm{N}^{02}$ & Réf \\
\hline Extraction & Gassoil & MJJ/t & 76,9 & 76,9 & {$[11]$} \\
\hline Séchage & Electricité & KWh/t & 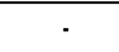 & 300 & [9] \\
\hline Concassage & Electricité & KWh & 0,57 & 0,57 & Estimation \\
\hline Broyage & Electricité & KWh & 10,90 & 10,90 & Estimation \\
\hline Tamisage & Electricité & $\mathrm{KWh}$ & 5,45 & 5,45 & Estimation \\
\hline $\begin{array}{c}\text { Calcination } \\
\text { flash }\end{array}$ & Gaz naturel & MJJt & 1894,7 & 1894,7 & [11] \\
\hline Stockage en sac & Papier kraft & $\mathrm{Kg}$ & 3 & 3 & Estimation \\
\hline
\end{tabular}

\subsubsection{Matières premières}

L'estimation de la composition minéralogique des sédiments de dragage du barrage est donnée par le tableau 2.

Table 2. Estimation minéralogique des sédiments de dragage du barrage de Fergoug.

\begin{tabular}{|c|c|c|c|}
\hline Minéraux & Composition & Classification & Sédiments \\
\hline Quartz & $\mathrm{SiO}_{2}$ & Oxyde de silicium & 42,62 \\
\hline Calcite & $\mathrm{CaCO}_{3}$ & Carbonate anhydre & 31,90 \\
\hline Dolomite & $\mathrm{CaMg}\left(\mathrm{CO}_{3}\right)_{2}$ & Carbonate anhydre & 12,06 \\
\hline Kaolinite & $\left(\mathrm{K}, \mathrm{H}_{3} \mathrm{O}\right)(\mathrm{Al}, \mathrm{Mg}, \mathrm{Fe})(\mathrm{Si}, \mathrm{Al})_{4} \mathrm{O}_{10}(\mathrm{OH})_{2}, \mathrm{H}_{2} \mathrm{O}$ & Phyllosilicate & 13,40 \\
\hline
\end{tabular}

\subsubsection{Extraction des sédiments (Engins) :}

Les émissions dans l'air sont présentées dans le tableau 3.

Table 3. Hypothèse de calcul d'après : Hugrel et Joumard, 2006[11].

\begin{tabular}{|c|c|c|c|}
\hline Emission dans l'air & Unité & Quantité & \multirow{9}{*}{$\begin{array}{c}\text { bibliographiques } \\
\text { [Hugrel et } \\
\text { Joumrad, 2006] }\end{array}$} \\
\hline $\mathrm{CO} 2$ & \multirow{8}{*}{$\mathrm{Kg} / \mathrm{t}$} & 5,599 & \\
\hline $\mathrm{CO}$ & & 0,016 & \\
\hline COGNM & & 0007 & \\
\hline $\mathrm{NOx}$ & & 0071 & \\
\hline $\mathrm{CH} 4$ & & 0 & \\
\hline Particules PM10 & & 0,004 & \\
\hline $\mathrm{N} 2 \mathrm{O}$ & & 0,001 & \\
\hline $\mathrm{H} 2 \mathrm{O}$ & & 0 & \\
\hline
\end{tabular}

\subsection{Méthodes de calcul des indicateurs d'impacts :}

Nous avons choisi la méthode CML baseline afin de réaliser l'évaluation des impacts. La réflexion sera basée sur 11 indicateurs qui sont : Epuisement des ressources, épuisement des ressources (combustibles fossiles), réchauffement climatique, destruction de la couche d'ozone, toxicité humaine, Ecotox aquatique d'eau douce, photochimique, acidification atmosphérique, eutrophisation [10].

\subsection{Evaluation des impacts environnementaux :}

Apres modélisation par le logiciel SimaPro 8.2.3 multi-user de l'école d'ingénieurs ICAM de Nantes, les figures suivantes représentent les impacts estimés pour la production d'une tonne de sédiments de dragage avec les deux scenarios envisagés. Cette modélisation nous permet d'avoir une première idée pour appréhender le développement d'un processus de production des sédiments de dragage.

a) Modélisation du scénario $\mathrm{N}^{\circ} 1$ de production des sédiments de dragage :

La figure 2 représente les impacts sur l'ensemble des indicateurs de la modélisation du scénario $\mathrm{N}^{\circ} 1$ de production des sédiments de dragage avec la méthode CML-IA.

La figure 2 nous permet de constater que la phase de calcination flash est la phase la plus impactante sur l'ensemble des indicateurs de la méthode CML-IA. Elle est suivie par le transport par tapis vibrant et le broyage qui sont aussi observés sur les huit catégories de dommages de la méthode CML-IA baseline. Ceci confirme les résultats faits lors de l'analyse de l'arborescence de la modélisation du scénario $\mathrm{N}^{\circ} 1$ de production des sédiments de dragage.

b) Modélisation du scénario $\mathrm{N}^{\circ} 2$ de production des sédiments de dragage :

La figure 3 représente les impacts sur l'ensemble des indicateurs de la modélisation du scénario $\mathrm{N}^{\circ} 2$ de production des sédiments de dragage avec la méthode CML-IA.

La figure nous permet de constater que la phase de calcination flash et le séchage sont les phases les plus impactantes sur l'ensemble des indicateurs de la méthode CML-IA.

\section{Comparaison et discussion des résultats :}

L'analyse du cycle de vie (ACV) des deux processus de production des sédiments de dragage permet d'envisager une comparaison environnementale des indicateurs d'impacts des deux procèdes envisagés afin d'optimiser le procédé le plus respectant de l'environnement. 
L'évaluation environnementale des deux procédés envisagés est représentée dans le tableau 4.

Le scénario $\mathrm{N}^{\circ} 1$ présente un excellent bilan environnemental dans l'ensemble des indicateurs de la méthode CML-IA par rapport au scénario $\mathrm{N}^{\circ} 2$.
La figure 4 représente l'histogramme de comparaison des impacts sur l'ensemble des indicateurs de la modélisation des deux scénarios de production des sédiments de dragage avec la méthode CML-IA.

a) scénario $\mathrm{N}^{\circ} 1$ :

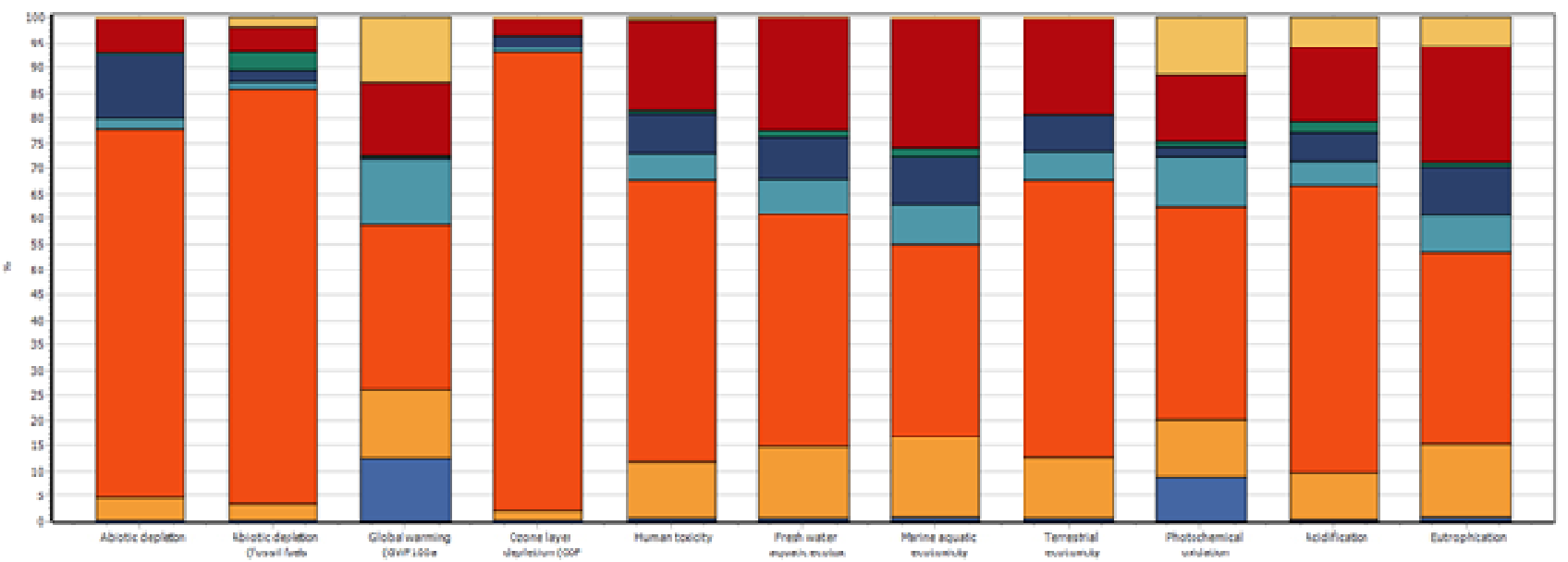

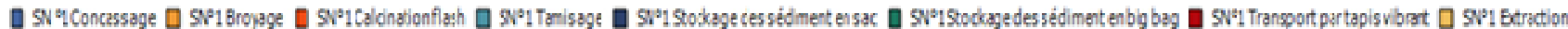

Fig. 2. Histogramme des impacts sur l'ensemble des indicateurs de la modélisation du scénario $\mathrm{N}^{\circ} 1$ de production des sédiments de dragage avec la méthode CML-IA

b) scénario $\mathrm{N}^{\circ} 2$ :

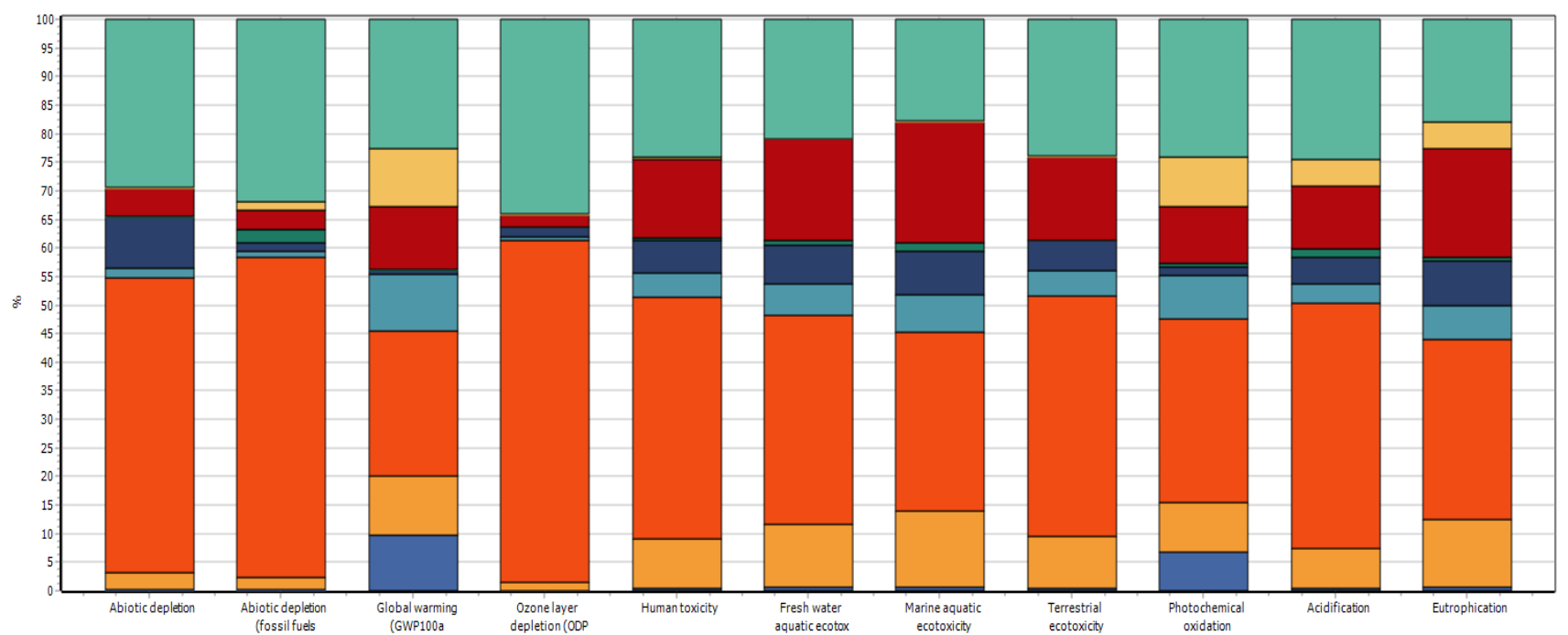

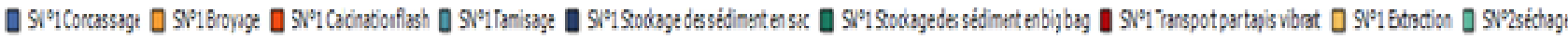

Fig. 3. Histogramme des impacts sur l'ensemble des indicateurs de la modélisation du scénario $\mathrm{N}^{\circ} 2$ de production des sédiments de dragage avec la méthode CML-IA 
Table 4. Indicateurs d'impacts environnementaux des deux scénarios de production des sédiments de dragage

\begin{tabular}{|c|c|c|c|}
\hline Catégorie d'impact & Unité & Scénario $N^{\circ} 1$ & Scénario $\mathrm{N}^{\circ} \mathrm{Z}$ \\
\hline Epuisement des ressources & Kg Sbeq & $7,56 \mathrm{E}-05$ & 0,000107 \\
\hline $\begin{array}{l}\text { Epuisement des ressources } \\
\text { (combustibles fossiles) }\end{array}$ & MJ & $2,91 E+03$ & $4,28 E+03$ \\
\hline Changement climatique & $\mathrm{KgCO} 2 \mathrm{eq}$ & 726 & 939 \\
\hline Destruction de la couche d'ozone & Kg CFC-11 eq & $1,95 \mathrm{E}-05$ & $2,97 E-05$ \\
\hline toxicité humaine & $\mathrm{Kg} \mathrm{1,4-DB}$ eq & 21,4 & 28,2 \\
\hline Ecotox aquatique d'eau douce & $\mathrm{Kg} \mathrm{1,4-DB} \mathrm{eq}$ & 19 & 24,1 \\
\hline Ecotoxicité aquatique marine & $K g 1,4-D B$ eq & $6,75 E-04$ & $8.22 \mathrm{E}+04$ \\
\hline Toxicité terrestre & Kg 1,4-DB eq & 0,133 & 0,175 \\
\hline Oxydation photochimique & $\mathrm{KgC2H4} \mathrm{eq}$ & 0,0644 & 0,0848 \\
\hline Acidification atmosphérique & $\mathrm{kgSOZ} \mathrm{eq}$ & 0,461 & 0,611 \\
\hline Eutrophisation & Kg PO4-- eq & 0,114 & 0,138 \\
\hline
\end{tabular}

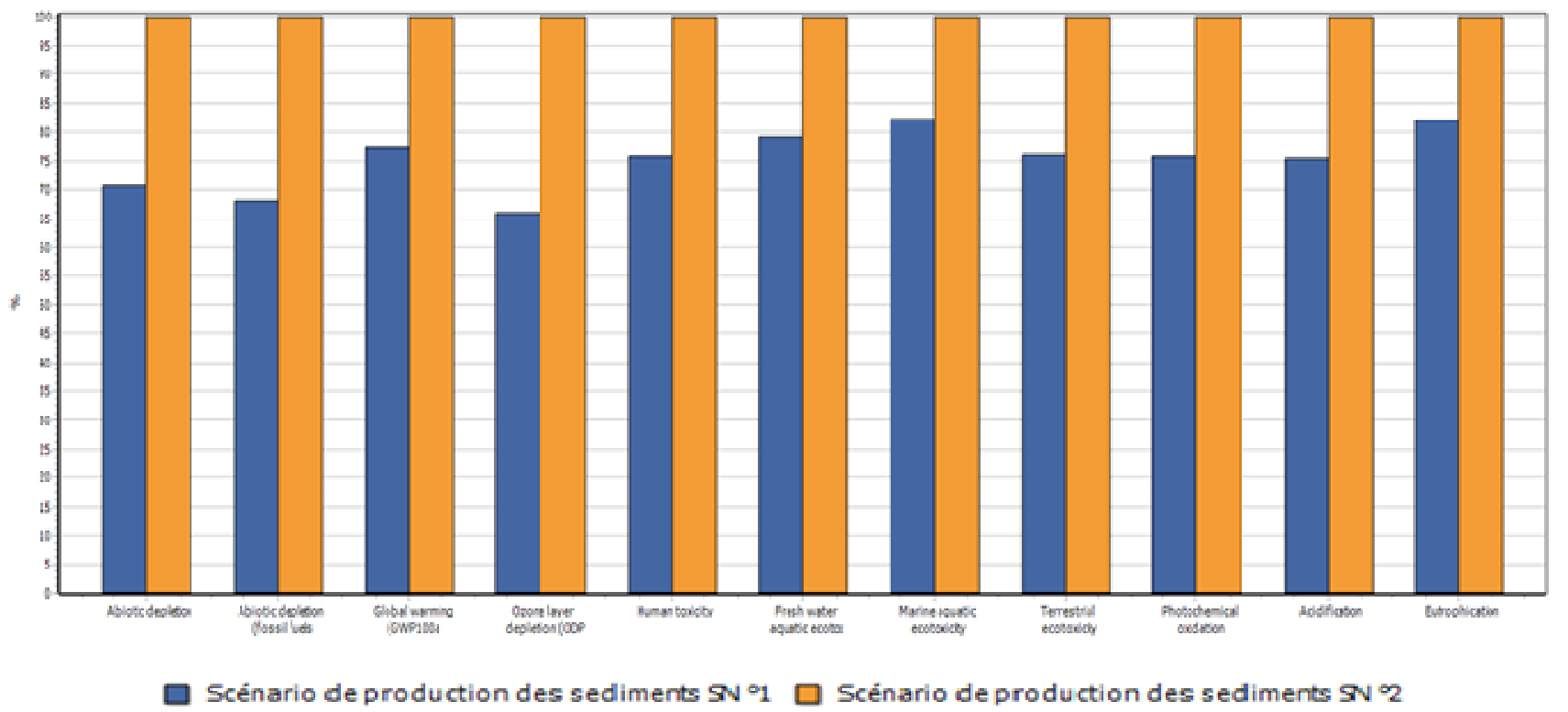

Fig. 4. Histogramme de comparaison des impacts sur l'ensemble des indicateurs de la modélisation des deux scénarios de production des sédiments de dragage avec la méthode CML-IA

Le scénario $\mathrm{N}^{\circ} 1$ de production des sédiments de dragage est le plus respectueux de l'environnement sur l'ensemble des indicateurs de la méthode CML-IA baseline offre un bénéfice moyen de l'ordre de $30 \%$ d'amélioration sur l'ensemble des indicateurs par rapport au scénario $\mathrm{N}^{\circ} 2$.

La figure 5 représente l'histogramme des processus à l'origine des impacts des deux scénarios $\mathrm{N}^{\circ} 1$ et $\mathrm{N}^{\circ} 2$ de production des sédiments de dragage.

Sur l'ensemble du processus de production des sédiments de dragage, on observe que la consommation du gaz naturel, avec un gain de $1,78 \mathrm{E}-5 \mathrm{~kg}$ CFC-11eq de la phase calcination flash, est le principal contributeur. Le second est la consommation de l'électricité avec un gain de 1,4E6 KgCFC-11eq dû à l'ensemble des opérations de traitement des sédiments de dragage. Le troisième est la consommation du papier Kraft avec un gain de 0,5E-6 KgCFC-11eq dû au stockage des matériaux.

La figure 6 représente l'histogramme des substances à l'origine des impacts des deux scénarios de production des sédiments de dragage.

Sur l'ensemble du processus de production des deux scénarios $\mathrm{N}^{\circ} 1$ et $\mathrm{N}^{\circ} 2$ des sédiments de dragage, on observe que les substances «Méthane, bromochlorodifluoro-, Halon $1211 »$, «Méthane, bromotrifluoro-,Halon1301 » et «Ethane, 1,2-dichloro1,1,2,2-tetrafluoro », sont les principaux contributeurs. Les autres couleurs correspondent à d'autres substances. 


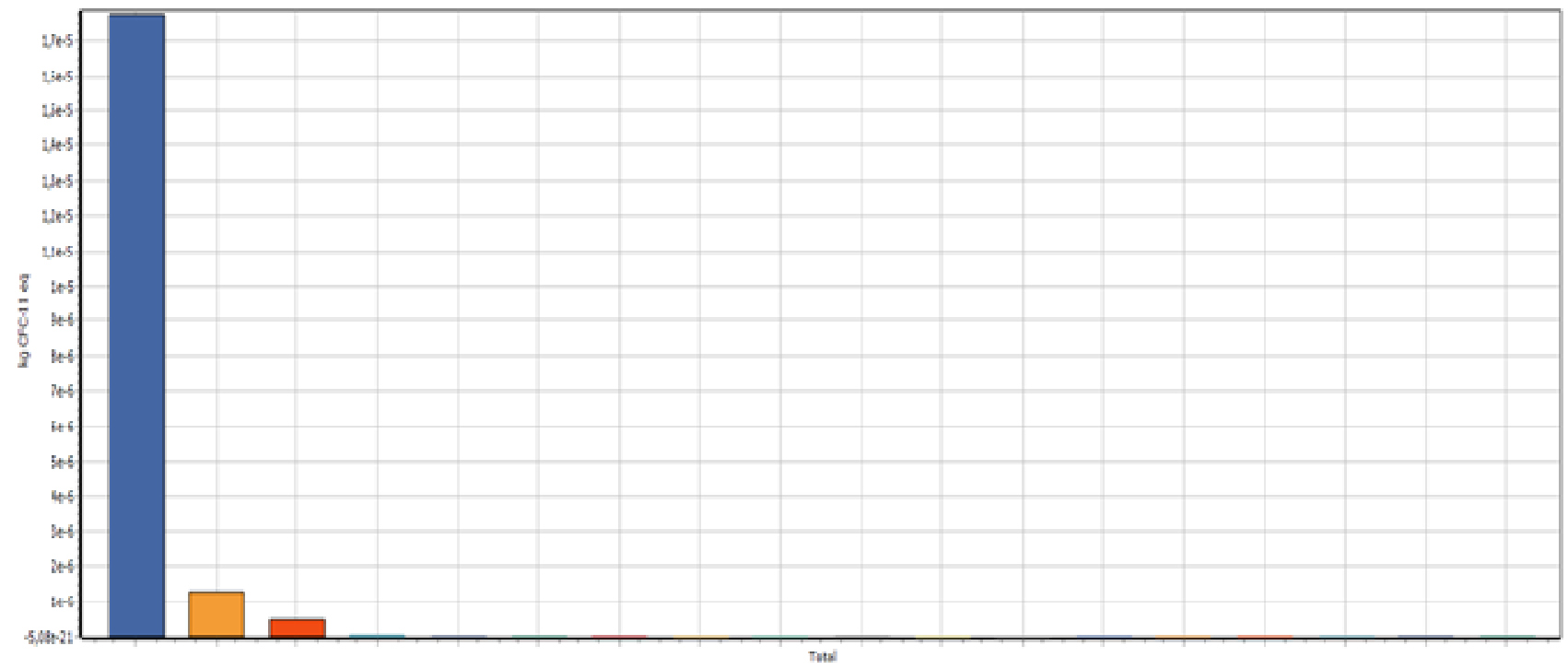

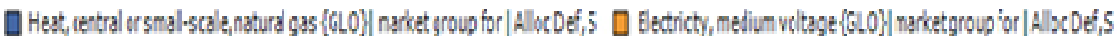

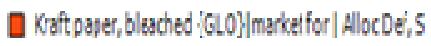

Fig. 5. Histogramme des processus à l'origine des impacts des deux scénarios $\mathrm{N}^{\circ} 1$ et $\mathrm{N}^{\circ} 2$ de production des sédiments de dragage

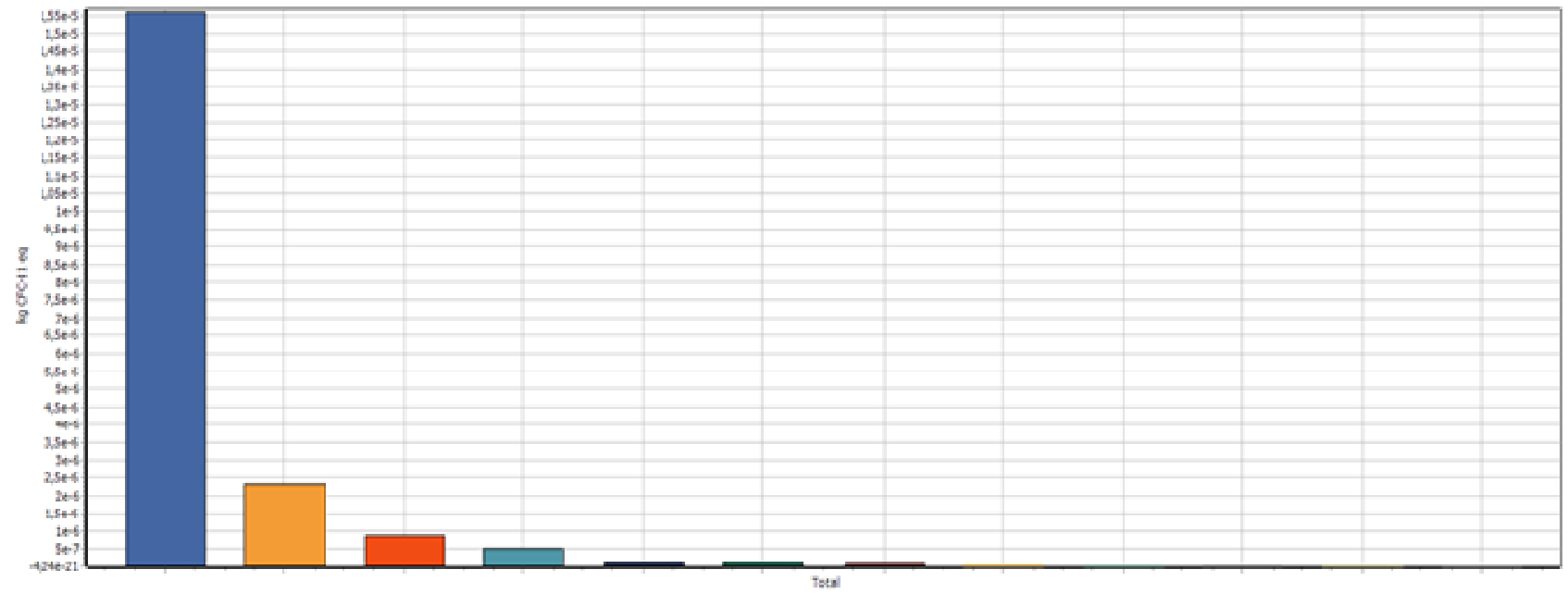

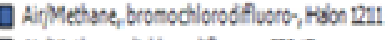

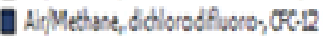

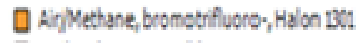

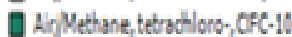

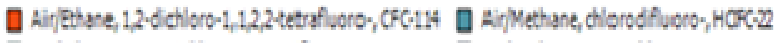

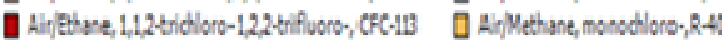

Fig. 6. Histogramme des substances à l'origine des impacts des deux scénarios $\mathrm{N}^{\circ} 1$ et $\mathrm{N}^{\circ} 2$ de production des sédiments de dragage

\section{Conclusion}

Les scénarios étudies ont porté sur le développement d'un nouveau procédé de production des sédiments de dragage en Algérie. Le scénario $\mathrm{N}^{\circ} 1$ consistait à utiliser les sédiments à l'état sec. Cette opération a demandé un temps de séchage à l'air libre assez important. Le scénario $\mathrm{N}^{\circ} 2$ a été fait sur des sédiments à l'état humide. Le temps de séchage sera plus réduit. Le choix se portera sur le scénario $\mathrm{N}^{\circ} 1$, les impacts environnementaux ont été plus faibles; d'où une nette amélioration sur les indicateurs environnementaux.

L'étape de calcination est la plus impactante sur l'environnement. Il Ya lieu d'envisager une recherche plus détaillée sur un nouveau procédé, avec une optimisation de consommation d'énergie.

\section{References}

1. F. KAZI AOUAL-BENSLAFA, (2014). Caractérisation des sédiments de dragage du barrage de Bouhanifia pour une réutilisation. XIIIème Journées nationales génie côtier, Dunkerque, (2014)

2. Y. SENHADJI, Evaluation de la vase de quatre barrages algériens, Conférence internationale des 
matériaux innovants \& leurs applications. Les 21et 22 avril 2016, Oujda-Maroc, (2016)

3. M.A. CHIKOUCHE M.A, Effet des ajouts à base d'argiles gréseuses et vaseuses sur les propriétés des matériaux cimentaires. Mémoire magister Université de M'sila, (2008)

4. A. SEMCHA, Valorisation des sédiments de dragage : Applications dans le BTP, cas du barrage de Fergoug. Thèse Université de Reims Champagne-Ardenne, (2008) 5. M. SILINE, Valorisation d'une argile gréseuse comme ajout pour la fabrication d'un ciment à faible impact environnemental. 31emes Rencontres de l'AUGC ENS, Cachan, (2013)

6. N.E. BOUHAMOU, Etude du comportement d'un béton autoplaçant à base de vase de dragage vis-à-vis du retrait. Colloque éco matériau, Montpellier, France, (214) 7. O. BELARIBI, Durabilité des bétons autoplaçants à base de vase et de pouzzolane. Thèse en cotutelle université de Mostaganem et l'université Cergy-pontoise, (2015)

8. ANBT, Agence nationale des barrages et transferts, (2015)

9. ADEME, DABEE, Département Industrie et Agriculture. Le séchage thermique ADEME, (2006)

10. J. OLIVIER, S. MYRIAM, Analyse du cycle de vie comprendre et réaliser un écobilan. 2eme édition, (2010)

\section{LABORATOIRE CENTRAL DES PONTS ET}

CHAUSSEES LCPC, Evaluation environnementale d'un procédé de fabrication de méta kaolin (calcination flash), (2010) 